\title{
Differentiation of PDX1 gene-modified human umbilical cord mesenchymal stem cells into insulin-producing cells in vitro
}

\author{
DONGMEI HE, JUAN WANG, YANGJUN GAO and YUAN ZHANG \\ Institute of Hematology, Medical College, Jinan University, Guangzhou 510632, P.R. China
}

Received June 13, 2011; Accepted July 18, 2011

DOI: 10.3892/ijmm.2011.774

\begin{abstract}
Mesenchymal stem cells (MSCs) have significant advantages over other stem cell types, and greater potential for immediate clinical application. MSCs would be an interesting cellular source for treatment of type 1 diabetes. In this study, MSCs from human umbilical cord were differentiated into functional insulin-producing cells in vitro by introduction of the pancreatic and duodenal homeobox factor 1 (PDX1) and in the presence of induction factors. The expressions of cell surface antigens were detected by flow cytometry. After induction in an adipogenic medium or an osteogenic medium, the cells were observed by Oil Red $\mathrm{O}$ staining and alkaline phosphatase staining. Recombinant adenovirus carrying the PDX1 gene was constructed and MSCs were infected by the recombinant adenovirus, then treated with several inducing factors for differentiation into islet $\beta$-like cells. The expression of the genes and protein related to islet $\beta$-cells was detected by immunocytochemistry, RT-PCR and Western blot analysis. Insulin and C-peptide secretion were assayed. Our results show that the morphology and immunophenotype of MSCs from human umbilical cord were similar to those present in human bone marrow. The MSCs could be induced to differentiate into osteocytes and adipocytes. After induction by recombined adenovirus vector with induction factors, MSCs were aggregated and presented islet-like bodies. Dithizone staining of these cells was positive. The genes' expression related to islet $\beta$-cells was found. After induction, insulin and C-peptide secretion in the supernatant were significantly increased. In conclusion, our results demonstrated that PDX1 gene-modified human umbilical cord mesenchymal stem cells could be differentiated into insulin-producing cells in vitro.
\end{abstract}

\section{Introduction}

Recent studies have demonstrated the feasibility of generating insulin-producing cells from stem cells and progenitor cells of various cellular sources (1-6). Despite their promising

Correspondence to: Dr Dongmei He, Institute of Hematology, Medical College, Jinan University, Guangzhou 510632, P.R. China E-mail: thedm@jnu.edu.cn

Key words: pancreatic and duodenal homeobox factor 1, mesenchymal stem cells, human, umbilical cord, $\beta$-like cells potential, it has proven to be difficult to obtain enough autologous adult stem cells. Meanwhile, the efficiency of inducing cell differentiation into the insulin-producing cells needs to be improved. Therefore, it is necessary to find other sources for cellular differentiation into insulin-secreting cells and to increase the efficiency of the differentiation. The expression of pancreatic and duodenal homeobox factor 1 (PDX1), which is the most important transcription factor in pancreatic differentiation, can switch on the differentiation and development of stem cells to pancreatic buds, and induce further differentiation $(7,8)$. Mesenchymal stem cells (MSCs) in the human umbilical cord are easily obtained when compared with embryonic and other stem cells. Recent studies described differentiation protocols to produce insulin-positive cells from embryonic stem cells $(1,9)$, hepatic stem cells $(10)$, bone marrow-derived cells $(11,12)$, umbilical cord blood cells $(13)$, human umbilical cord cells (14), or genetically modified stem cells using several different pancreas-specific transcription factor genes $(2,3,15)$. However, the potential of human umbilical cord MSCs by genetic manipulation combined with inducing factors to form insulin-producing cells has not yet been evaluated. Therefore, it is interesting to construct a genetic expression vector carrying PDX1 and to investigate the effect of PDX1 combined with inducing factors on the differentiation of MSCs into insulin-producing cells. In this report, we present a multistep differentiation protocol to generate insulin-producing cells. Our results show that MSCs from human umbilical cord can be induced to differentiate into islet $\beta$-like cells, which can secrete insulin and C-peptide, and are sensitive to stimulation with glucose.

\section{Materials and methods}

Construction of pAdxsi-CMV-PDX1. The full-length human PDX1 cDNA was kindly provided by Dr Tang Xiaolong (Institute of Hematology, Medical College, Jinan University). The human PDX1 gene was cloned into the pUC57 plasmid (Gibco-Invitrogen, USA). The PDX1 was cut out of the pUC57-PDX1 by XhoI enzyme and then the PDX1 gene was integrated into Shuttle-CMV plasmid (Gibco-Invitrogen) with green fluorescence protein (GFP) gene. The PDX1 gene from pShuttle-GFP-CMV-PDX1 was transferred into adenoviral vector (pAdxsi) (Gibco-Invitrogen) and linearized with I-CeuI + I-SceI enzyme. All sequences were confirmed by DNA sequencing. The confirmed recombinant adenoviral 
construct pAdxsi-CMV-PDX1 was transfected into the 293 cell line using Lipofectamine 2000 (Gibco-Invitrogen). After 3 days of infection, the viruses were harvested. A virus titer was determined as a multiplicity of infection (MOI) by a standard tissue culture infectious dose (TCID50) assay (refer to the AdEasy ${ }^{\mathrm{TM}}$ Adenoviral Vector System method, Stratagene Products, USA). The harvested viruses were filtered through a $0.45 \mu \mathrm{m}$ filter membrane (Millipore) and concentrated by a Spin Column (Millipore).

Isolation of MSCs and analysis of MSC surface glycoprotein. The use of human umbilical cord was approved by The First Affiliated Hospital of Jinan University. All procedures were conducted in accordance with the guidelines of the Medical Ethics Committee of the Health Bureau of the Guangdong Province, China. Umbilical cord specimens were carefully dissected. The harvested pieces of tissue were washed several times in phosphate-buffered saline (PBS) and then mechanically minced and enzymatically digested with $0.1 \%$ collagenase II (Gibco-Invitrogen) for approximately $30 \mathrm{~min}$ at $37^{\circ} \mathrm{C}$. Digested tissue was filtered twice through a $100 \mu \mathrm{m}$ nylon membrane to eliminate undigested fragments. The cell suspension was collected by Ficoll-Hypaque gradient fractionation at $1000 \mathrm{r} / \mathrm{min}$ for $5 \mathrm{~min}$. The mononuclear cells were suspended in $5 \mathrm{ml}$ Dulbecco's modified Eagle's medium/ F12 (DMEM/F12) (Gibco-Invitrogen) supplemented with $10 \%$ fetal bovine serum (FBS) (PAA Laboratories, Austria), cultured at $37^{\circ} \mathrm{C}$ in $5 \% \mathrm{CO}_{2}$ in fully humidified air. When the adherent cells reached $80 \%$ confluence, $50 \%$ of the medium was replaced with fresh medium, followed by media changes every 3-4 days. Cultured cells were harvested after four cell passages. Cells were incubated with respective fluorochromeconjugated antibodies for cell surface glycoprotein: CD106, CD45, CD34, CD29, CD44, CD14, CD31 (BD Biosciences, San Diego, CA) and HLA-DR (Immunotech, Marseille, France). These are commonly used for the positive and negative detection of MSCs. Cells were incubated with antibody for $30 \mathrm{~min}$ on ice and immediately analyzed by flow cytometry (FACScan, BD Biosciences).

Osteogenic and adipogenic differentiation of MSCs. Cultured MSCs were assessed at the fourth passages. Osteogenic differentiation was assessed by incubating the cells using DMEM/ F12 with $10 \%$ FBS, $0.1 \mu \mathrm{mol} / 1$ dexamethasone, $10 \mathrm{mmol} / 1$ $\beta$-glycerophosphate and $50 \mu \mathrm{mol} / 1$ ascorbate acid (Sigma, St. Louis, MO). Osteoblasts were identified by immunocytochemical staining with alkaline phosphatase. Adipogenic differentiation was assessed by using DMEM/F12 supplemented with $100 \mathrm{ml} / \mathrm{l} \mathrm{FBS}, 1 \mu \mathrm{mol} / \mathrm{l}$ dexamethasone and $5 \mathrm{U} / \mathrm{ml}$ insulin (Sigma). Adipogenic differentiation cells were determined by the cellular accumulation of neutral lipid vacuoles stained with Oil Red O (Sigma).

The pAdxsi-CMV-PDX1 infection and induction of MSCs. Our study was divided into four groups and three stages. The four groups included the MSCs control group, pAdxsi-CMVPDX1 + induction factor group, vector group + induction factor group, and induction factor group. To optimize the induction protocol, differentiation of MSCs was carried out in three stages. In stage 1, undifferentiated MSCs were infected
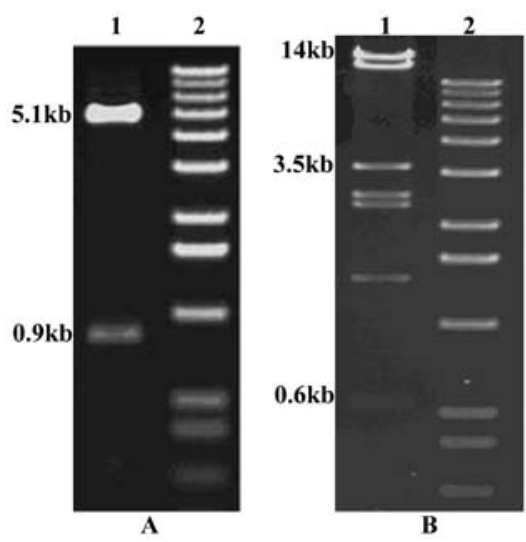

Figure 1. Identification of recombinant adenoviruses pAdxsi-GFP-PDX1 by digestion. (A) pShuttle-GFP-CMV-PDX1 was digested by $B g l$ II (lane 1); (B) pAdxsi-GFP-PDX1 was digested by XhoI (lane 1); lane 2 marker showing bands of $8,7,6,5,4,3,2,1.6$ or $1 \mathrm{~kb}$ and 517,396 and $230 \mathrm{bp}$.

with pAdxsi-CMV-PDX1 and then cultured for 7 days. In stage 2, cells were cultured in 2\% FBS/DMEM/F12 medium and supplemented with $100 \mathrm{ng} / \mathrm{ml}$ epidermal growth factor (EGF) and 2\% B27 (Sigma) for 3 days. In stage 3, cells were exposed to a cocktail of $10 \mathrm{ng} / \mathrm{ml}$ glucagons-like peptide- 1 (GLP-1), $10 \mathrm{ng} / \mathrm{ml}$ betacellulin, $10 \mathrm{ng} / \mathrm{ml}$ hepatocyte growth factor (HGF), $10 \mathrm{mmol} / 1$ nicotinamide, $2 \% \mathrm{~B} 27,0.1 \mathrm{mmol} / 1$ $\beta$-mercaptoethanol (Sigma) for 7 days.

Dithizone staining. The induction cells and the uninduction cells were collected and washed in PBS. Then, $0.1 \mathrm{ml} \mathrm{0.05 \%}$ (mg/l) Dithizone (Sigma) was added, stained and incubated at $37^{\circ} \mathrm{C}$ for $15 \mathrm{~min}$. Then cells were examined under a microscope.

Immunocytochemistry and immunofluorescence analysis. The induced and uninduced MSCs were collected. After washing in PBS, cells were fixed with $4 \%$ paraformaldehyde (pH 7.4) for 10 min and rinsed again with PBS. The cells were permeabilized with $0.1 \%$ Tween-20 in PBS for 5 min and 3\% bovine serum albumin (BSA, Sigma) for $30 \mathrm{~min}$. Then cells were incubated overnight at $4^{\circ} \mathrm{C}$ with the primary anti-human antibody, 1:100 (mouse anti-human PDX1/IgG, insulin/IgG, rabbit anti-human C-peptide/IgG, Abcam, USA). Cells were washed twice in PBS and incubated for $1.5 \mathrm{~h}$ with HRP-antimouse/rabbit IgG, 1:300 (Sigma) or fluorescence-conjugated second antibody, 1:300 (Dako, Denmark, Cy3, Cy5 and FITC). DNA-specific fluorescent dye Hoechst 33258 (Abcam) stain was used to detect cell nuclei. The cells were photographed and visualized under a microscope (Leica, Germany).

Reverse transcription-polymerase chain reaction. RNA was extracted from cultured cells using TRIzol extraction kit (Invitrogen). Synthesis of cDNA was performed using AMV reverse transcriptase (Takara, Japan) following the manufacturer's instruction. The primer sequence was as follow, GAPDH, forward, 5'-AGAAGGCTGGGGCTCATTTG-3' and reverse, 5'-AGGGGCCATCCACAGTCTTC-3' '258 bp; PDX1, forward, 5'-GGATGAAGTCTACCAAAGCTCACGC-3' and reverse, 5'-CCAGATCTTGATGTGTCTCTCGGTC-3', 230 bp; insulin, forward, 5'-AACCAACACCTGTGCGGCTCA-3' and reverse, 5'-TGCCTGCGGGCTGCGTCTA-3', 271 bp; Glut-2, 


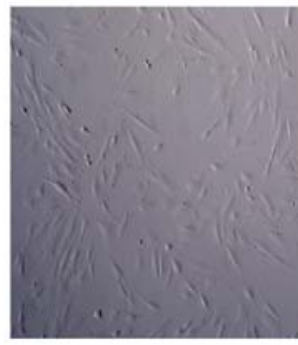

A

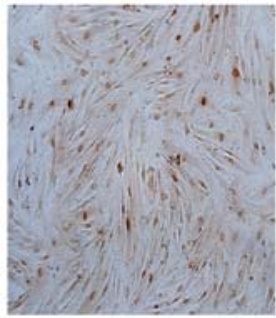

E

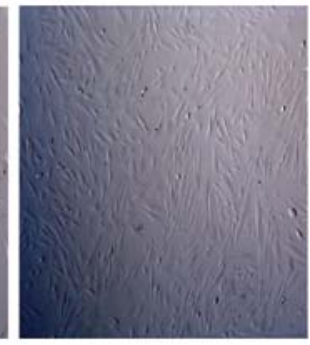

$\mathbf{B}$

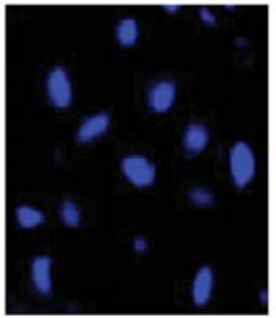

$\mathbf{F}$

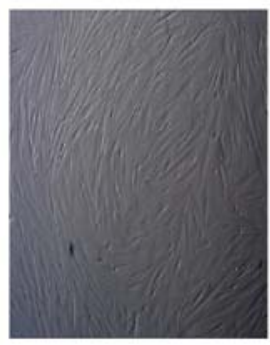

C

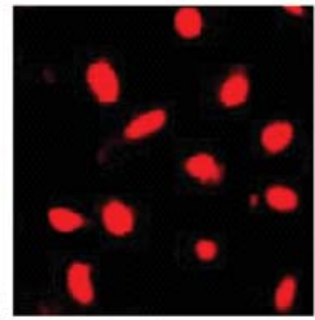

G

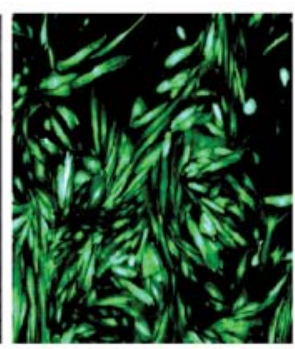

D

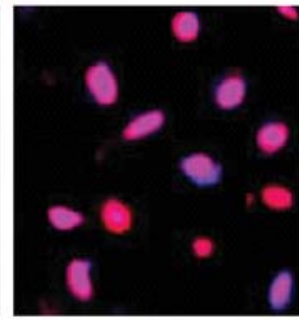

H

Figure 2. Morphology of MSCs in culture and the expression of PDX1 in infected MSCs. MSCs isolated from human umbilical cord in culture for (A) 7 and (B) 15 days or (C) after four cell passages. (D) GFP expression after pAdxsi-CMV-PDX1 infection at $24 \mathrm{~h},(\mathrm{E}) \mathrm{PDX} 1 \mathrm{expression}$ by immunocytochemistry and (G) immumofluorescence $48 \mathrm{~h}$ (Cy3, red) after pAdxsi-CMV-PDX1 infection; (F) MSCs stained to display the nuclear label Hoechst 33258 (blue). (H) Merged nuclear images of double staining with PDX1 (Cy3) and Hoechst 33258; , agnification: A, B and C, x100; D and E, x200; F-H, x400.

forward, 5'-GCGAATAAACAGGCAGGAGC-3' and reverse, 5'-GCTGGATACAGACAGGGACC-3', 392 bp. The GAPDH gene served as an internal control. Polymerase chain reaction (PCR) amplification was performed according to the following cycling parameters: $95^{\circ} \mathrm{C} 30 \mathrm{sec}, 60^{\circ} \mathrm{C} 30 \mathrm{sec}, 72^{\circ} \mathrm{C} 60 \mathrm{sec}$ for 40 cycles. PCR products were separated by electrophoresis on a $1 \%$ agarose gel containing ethidium bromide.

Western blot analysis. Cells were washed with PBS $(10 \mathrm{mM}$, $\mathrm{pH} 7.4)$ and incubated in $200 \mathrm{ml}$ of cell lysis buffer $(50 \mathrm{mmol} / \mathrm{l}$ Tris- $\mathrm{HCl}, 5 \mathrm{mmol} / \mathrm{l}$ EDTA, $25 \%$ sucrose, $0.6 \mathrm{ml}$ of $100 \mathrm{mg} / \mathrm{ml}$ phenylmethylsulphonyl fluoride, $2.4 \mathrm{ml}$ 2-mercaptoethanol) on ice for $30 \mathrm{~min}$, and centrifuged at $13,000 \mathrm{x} \mathrm{g}$ for $45 \mathrm{~min}$ at $4^{\circ} \mathrm{C}$. Proteins of the cell lysates were separated by $8 \%$ SDS polyacrylamide gel electrophoresis and electrotransferred to nitrocellulose membranes (Gibco-BRL, USA). The blot was put in blocking buffer (10\% non-fat dry milk, $1 \%$ Tween-20; in $20 \mathrm{mM}$ Tris-buffered saline, $\mathrm{pH}$ 7.5) for $1 \mathrm{~h}$ at room temperature and incubated with appropriate anti-human primary antibody, 1:100 (Abcam, mouse anti-human PDX1/IgG, insulin/IgG, GAPDH, rabbit anti-human C-peptide/IgG) in blocking buffer overnight at $4{ }^{\circ} \mathrm{C}$. The blots were incubated with anti-mouse/ rabbit horseradish peroxidase-conjugated secondary antibody (1:200) for $1 \mathrm{~h}$ and detected by chemiluminescence using ECL Hyperfilm.

Insulin and C-peptide secretion detection. After the induction was completed, the culture supernatants were collected. For the in vitro insulin secretion assay after hyperglycemic stimulation, cell clusters were collected, washed with PBS 5 times to prevent insulin assay cross-reaction with the medium. After clusters were stimulated with $25 \mathrm{mM}$ glucose, supernatants were harvested for measurement with a human insulin RIA kit and an ultrasensitive human C-peptide chemiluminescence kit (Abbott AxSym System, USA).
Statistical analysis. The data quantitated are expressed as mean \pm SE. The results were analyzed by one-way ANOVA. $\mathrm{P}<0.05$ was considered statistically significant.

\section{Results}

Identification of pShuttle-CMV-PDX1 and pAdxsi-CMV$P D X 1$. After the pShuttle-CMV-PDX1 plasmid was cut by $B g l$ II, two bands of 0.9 and $5.1 \mathrm{~kb}$ were detected. The pAdxsiCMV-PDX1 plasmid was cut by XhoI enzyme and the products observed were seven bands of 14, 11.8, 3.5, 2.66, 2.47, 1.45 and $0.6 \mathrm{~kb}$. These results indicate that PDX1 cDNA is inserted into the pAdxsi vector and is correctly linked (Fig. 1). The destination gene inserted into pShuttle-CMV-PDX1 vector was confirmed to be completely identical with the sequence of PDX1 (0.87 kb) cDNA.

The expression of MSC markers. Immunophenotype analysis revealed MSCs from human umbilical cord to be positive for CD44 (98.7\%) and CD29 (99.6\%). At the same time, MSCs were low for CD14 (2.8\%), CD45 (2.3\%), CD34 (1.6\%), CD106 (1.9\%), CD31 (2.5\%) and HLA-DR (0.68\%).

The induction of differentiation into osteocytes and adipocytes. To verify that MSCs from human umbilical cord are multipotent, we analyzed their ability to differentiate into osteocytes and adipocytes. After 28 days of induction, it was shown that the MSCs differentiated into osteocytes and adipocytes by the increase of alkaline phosphatase and Oil Red $\mathrm{O}$ staining, respectively, suggesting that MSCs could be induced to differentiate into osteocytes and adipocytes (not shown).

Morphological characterization of MSCs and the expression of PDX1. Under an inverted microscope, after four cell passages, undifferentiated MSCs were observed to be very flat, 


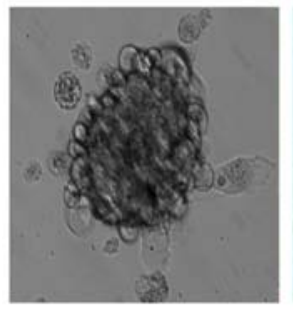

A

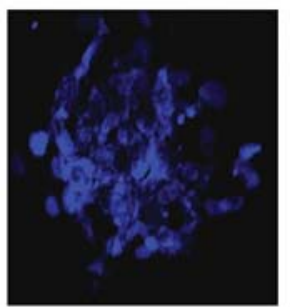

E

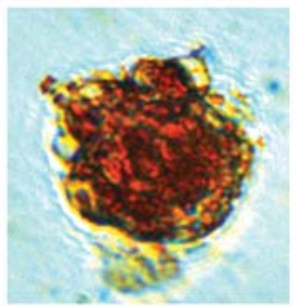

B

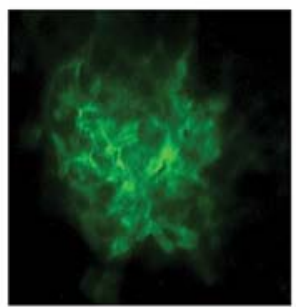

F

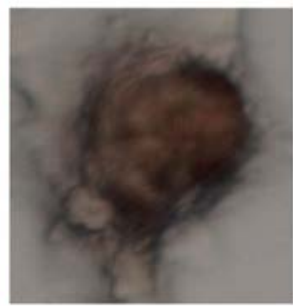

C

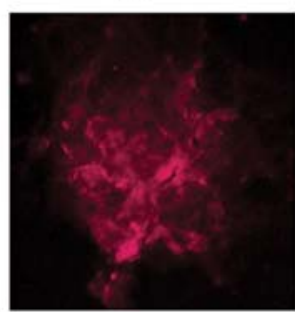

G

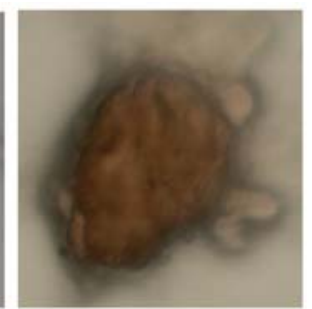

D

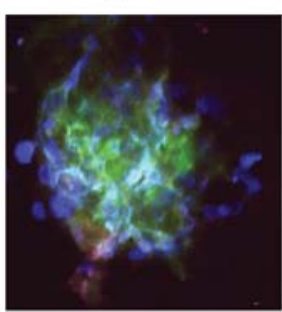

H

Figure 3. Morphological changes and insulin and C-peptide expression in inducing MSCs. (A) The morphological change of islet-like clusters after induction (x400); (B) morphological, positive change of dithizone staining of islet-like clusters (x400); (C) insulin and (D) C-peptide immunocytochemistry of islet-like clusters (x400); (E) Hochest 33258 for nucleus (x400); (F and G) C-peptide (FITC) and insulin (Cy5) expression in islet-like cluster after induction (x400); (H) A merged image from $\mathrm{E}, \mathrm{F}$ and $\mathrm{G}$ images $(\mathrm{x} 400)$

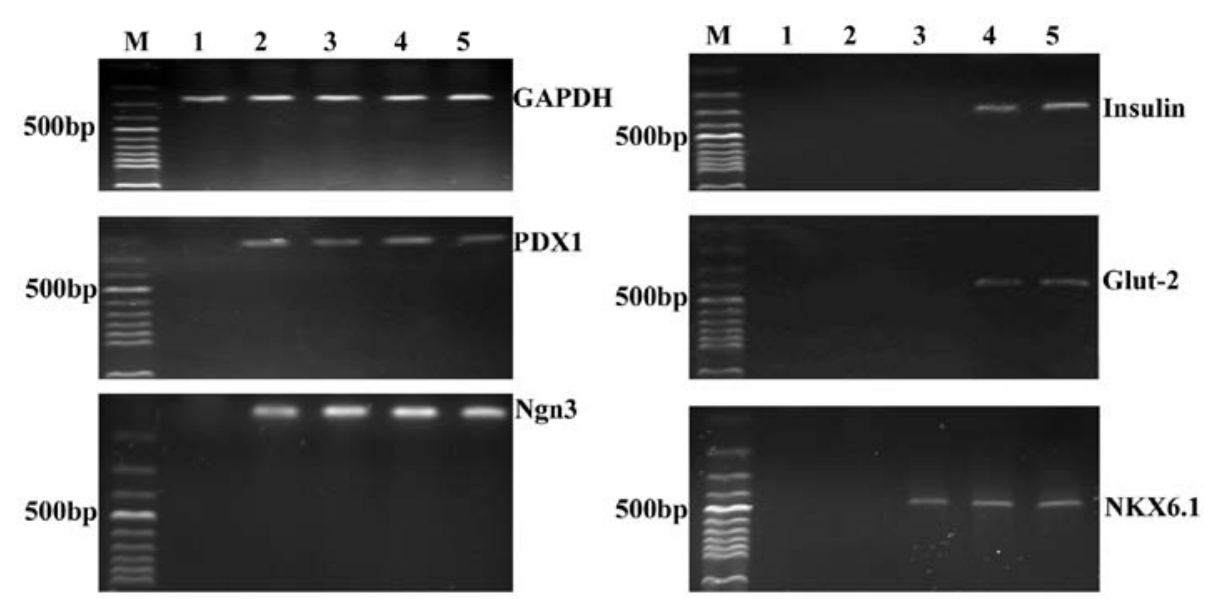

Figure 4. Expression of insulin-related genes in MSCs induced by induction factors and infected with pAdxsi-CMV-PDX1. Lanes 1-5, expression after induction at Days 0, 7, 10 and 17 and on Day 17 day with high glucose. M, 100 bp DNA marker.

symmetrical, and spindle-shaped (Fig. 2A, B and C). To observe the adenovirus infection efficiency in MSCs, the expression of GFP protein was determined under an inverted fluorescence microscope. GFP protein was expressed at a high level after pAdxsi-CMV-PDX1 infection at $24 \mathrm{~h}$ (Fig. 2D). After pAdxsiCMV-PDX1 transfection at $24 \mathrm{~h}, \mathrm{PDX} 1$ protein was detected by immunocytochemistry and immumofluorescence, and was localized in the nucleus. (Fig. 2E-H). The cells in the vector control transfection did not express PDX1 protein (not shown).

Morphological changes and protein expression of induced MSCs. The MSCs appearance began to change after infection of pAdxsi-CMV-PDX1 with induction factor. The shape of the cells became round and cells gathered gradually to form islet-like clusters (Fig. 3A). The zinc-chelating agent Dithizone is known to selectively stain pancreatic $\beta$-cells, which contain relatively high levels of zinc, crimson red (15). We previously demonstrated that Dithizone stain could also be applied for the detection of MSC-derived insulin-producing cells. In our study, Dithizone-positive cellular clusters were identified by the Dithizone staining, which suggests that the induced cells contain substantial amounts of zinc (Fig. 3B). Immunocytochemical staining indicated that insulin and C-peptide were expressed mainly in stage 3 of MSCs differentiation (Fig. 3C and D). Immunofluorescence analysis demonstrated the expression of insulin (Fig. 3G) in the second stage and of C-peptide in the third stage (Fig. 3F). The cells in all three control groups showed a small change in shape and no expression of insulin and C-peptide.

RT-PCR and Western blot analysis. PAdxsi-CMV-PDX1transfected MSCs expressed the transcription factor PDX1 at $24 \mathrm{~h}$ and until 3 weeks (not shown). As shown in Fig. 4, expression of the $\beta$-cell related gene Ngn3 was first detected on Day 7. Expression of the insulin gene was first observed in the second stage. In this stage, the $\beta$-cell-related gene Glut- 2 


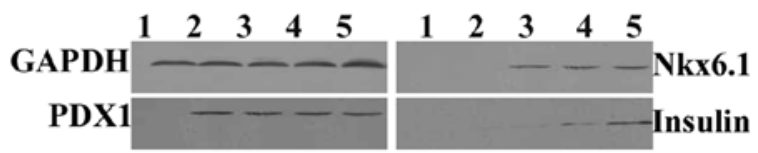

Figure 5. Insulin protein expression in induced MSCs. Lanes 1-5, protein expression after induction on Days 0, 7, 10 and 17 and on Day 17 with the addition of high glucose. M, 100 bp DNA marker.

was also detected. When the induction cells were stimulated with high glucose, the expression of insulin and of the Glut-2 gene increased. The PDX1 protein was detected at the second day after infection with recombinant adenovirus and was stable for 21 days (Fig. 5). Protein expression of NKX6.1 and insulin was observed on Day 10 of induction. The expression of insulin was increased on Day 17 and in the stage with the hyperglycemic stimulation. The cells without transfection by pAdxsi-CMV-PDX1 or with only the addition of induction factors did not express these genes (not shown).

Insulin and C-peptide detection by chemiluminescence. The insulin level was maintained at $4.1 \pm 5.9 \mathrm{mU} / 1$ prior to induction. After induction on Days 10 and 17, a very high insulin secretion in the supernatant was detected and the levels reached $255.8 \pm 24.9$ and $473.1 \pm 51.5 \mathrm{mU} / 1$ respectively, a significant difference in comparison with all three control groups $(\mathrm{P}<0.05)$. An especially high insulin concentration was observed when glucose (25 mmol/l) (Fig. 6) was added to the medium on Day 17. Insulin levels reached 3539.8 $\pm 245.1 \mathrm{mU} / \mathrm{l}$. However, no significant differences in insulin concentration were observed in the control groups. On Days 10 and 17 of induction, the levels of C-peptide secretion were $0.13 \pm 0.04$ and $1.61 \pm 0.41 \mathrm{ng} / \mathrm{ml}$. The levels of C-peptide secretion were $3.72 \pm 1.52 \mathrm{ng} / \mathrm{ml}$ at $1 \mathrm{~h}$ after stimulation with $25 \mathrm{mmol} / \mathrm{l}$ glucose. The cells in all three control groups did not release a measurable amount of C-peptide into the medium. These results indicate that the induced cells generated by our protocol were able to secrete insulin and C-peptide in response to a high concentration of glucose.

\section{Discussion}

Our results indicate that MSCs from human umbilical cord were similar to those present in adult human bone marrow. Their morphology and immunophenotype were similar to those of adult bone marrow MSCs. Like human bone marrowderived MSCs, human umbilical cord MSCs also expresses a large number of adhesion molecules, including CD29 and CD44. In order to prove that what we isolated from human umbilical cord was MSCs, the isolated cells were also induced to differentiate into adipocytes and osteoblasts. Our results suggest that the isolated cells had the characteristics of MSCs.

Our results show that the expression of PDX1 and inducing factors, such as EGF, B27 and GLP-1, could induce MSCs from human umbilical cord to form pancreatic islet-like cell clusters in vitro. These islet-like cell clusters were shown to release human insulin and C-peptide. Insulin gene transcription-regulating mechanism of pancreatic $\beta$-cells is extremely complicated. PDX1 has been proven to play an important role in regulating insulin gene expression and in the activation of the differentiation process of pancreas precursor cells to

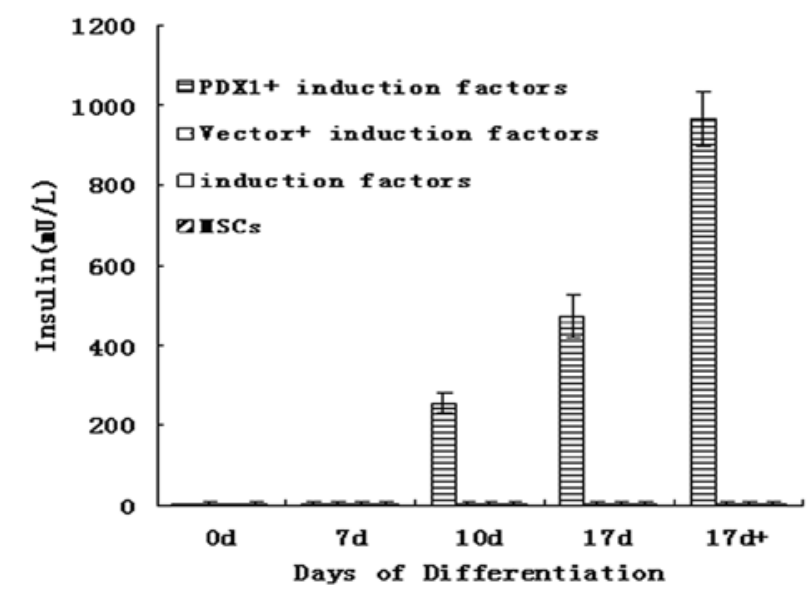

Figure 6. Secretion of insulin from MSCs after induction at different times. Values represent the mean $\pm \mathrm{SE}$ of five independent experiments. Cell supernatants were collected for insulin release analysis at Days 0, 7, 10 and 17 of induction. $17 \mathrm{~d}^{+}$, at Day 17 of induction glucose $(25 \mathrm{mmol} / \mathrm{l})$ was added to the medium for an additional one hour.

pancreas cells, including exocrine and endocrine cells. Our results show that PDX1 or the induction factors alone failed to induce cell differentiation. PDX1 combined with a series of induction factors can control and limit the differentiation of MSCs to $\beta$-like cells. These insulin-producing cells are responsive under the medium containing high glucose. It suggested the combination of PDX1 with induction factors may be an effective method to induce the MSCs into insulinproducing cells. Accordingly, our findings suggest that the synergistic effect of PDX1 and induction factors is beneficial to the differentiation of $\beta$-cells.

In the present study, in order to further optimize the protocol of induction, besides transferring of PDX1, we also chose to use a combination of various factors, including B27, GLP-1, human betacellulin, HGF and $\beta$-mercaptoethanol, as well as HGF and EGF, in which EGF can induced stem cell differentiation to pancreatic precursor cells called nestin-positive cells $(1,16)$. The induction factors GLP-1, human betacellulin, HGF and $\beta$-mercaptoethanol also influence $\beta$-cell development and functions. Among these factors, GLP-1 promotes fetal pancreatic precursor cell differentiation to $\beta$-cells through interaction with the receptor GLP-1R $(17,18)$. Nicotinic amide combined with betacellulin promotes the differentiation from stem cells to $\beta$-cells. Furthermore, betacellulin has the ability of promoting the differentiation of $\beta$-cells and speeding up the formation of pancreatic cell clusters (19-21). In addition, nicotinamide promotes the differentiation of fetal pancreatic precursor cells and increases the amount of $\beta$-cells $(22,23)$. $\beta$-mercaptoethanol is defined as a more strong oxidant inducing the change of cellular morphology from fusiform cells to round or nearly round cells through the variation of the cellular oxidation condition. These events are favorable for cell aggregation (24). The association of these factors provides a microenvironment that favors the maturation and function of $\beta$-cells. After 3 days induction, the MSCs changed to a round type, gathered and formed the pancreatic corpuscular.

In our study, a sequential expression of the key transcription factors linked to pancreas development emerged. Besides PDX1, the sequential appearance of Ngn3 and Nkx6.1, insulin 
and Glut-2 suggests that the induction of $\beta$-like cells followed a progression similar to that of in vivo pancreas development (25). Our finding is consistent with the reports in which PDX1 expression results in increased Ngn3 expression $(7,26)$, in which PDX1, Ngn3 and Nkx6.1 are important for differentiation of pancreatic endocrine cells and especially of $\beta$-cells $(27,28)$.

In summary, our findings indicated that MSCs from human umbilical cord could be induced to differentiate into mature islet-like cell clusters, which possess insulin-producing ability in vitro. Therefore, MSCs from the human umbilical cord have the potential to become an excellent candidate in $\beta$-cell replacement therapy of diabetes. However, the functional stability of the islet-like cells in vivo needs further investigation.

\section{Acknowledgements}

We thank Dr Tang Xiaolong and Dr Lu Yan, Institute of Hematology, Medical College, Jinan University, Guangzhou, for kindly providing valuable technical support, advice and vector. This study was supported by a grant from the Overseas Chinese Affairs Office of the State Council Key Discipline Construction Fund (no. 51205002), China.

\section{References}

1. Jiang J, Au M, Lu K, Eshpeter A, Korbutt G, Fisk G and Majumdar AS: Generation of insulin-producing islet-like clusters from human embryonic stem cells. Stem Cells 25: 1940-1953, 2007.

2. Karnieli O, Izhar-Prato Y, Bulvik S and Efrat S: Generation of insulin-producing cells from human bone marrow mesenchymal stem cells by genetic manipulation. Stem Cells 25: 2837-2844, 2007.

3. Noguchi H, Xu G, Matsumoto S and Kaneto H, Kobayashi N, Bonner-Weir S and Hayashi S: Induction of pancreatic stem/ progenitor cells into insulin-producing cells by adenoviral-mediated gene transfer technology. Cell Transplant 15: 929-938, 2006.

4. Sun NZ and Ji HS: In vitro differentiation of human placentaderived adherent cells into insulin-producing cells. J Int Med Res 37: 400-406, 2009.

5. Tateishi K, He J, Taranova O, Liang G, D'Alessio AC and Zhang Y: Generation of insulin-secreting islet-like clusters from human skin fibroblasts. J Biol Chem 283: 31601-31607, 2008.

6. Yang JH, Lee SH, Heo YT, Uhm SJ and Lee HT: Generation of insulin-producing cells from gnotobiotic porcine skin-derived stem cells. Biochem Biophys Res Commun 397: 679-684, 2010.

7. Oliver-Krasinski JM, Kasner MT, Yang J, Crutchlow MF, Rustgi AK, Kaestner KH and Stoffers DA: The diabetes gene Pdx1 regulates the transcriptional network of pancreatic endocrine progenitor cells in mice. J Clin Invest 119: 1888-1898, 2009.

8. Wang H, Maechler P, Ritz-Laser B, Hagenfeldt KA, Ishihara H, Philippe $\mathrm{J}$ and Wollheim CB: Pdx1 level defines pancreatic gene expression pattern and cell lineage differentiation. J Biol Chem 276: 25279-25286, 2001.

9. Kroon E, Martinson LA, Kadoya K, et al: Pancreatic endoderm derived from human embryonic stem cells generates glucoseresponsive insulin-secreting cells in vivo. Nat Biotechnol 26: 443-452, 2008

10. Khan AA, Rajendraprasad A, Parveen $\mathrm{N}$, et al: In vitro insulin production and analysis of pancreatic transcription factors in induced human hepatic progenitor cells. Diabetes Technol Ther 12: $373-378,2010$
11. Zhang Y, Shen W, Hua J, et al: Pancreatic islet-like clusters from bone marrow mesenchymal stem cells of human first-trimester abortus can cure streptozocin-induced mouse diabetes. Rejuvenation Res 13: 695-706, 2010.

12. Tayaramma T, Ma B, Rohde M and Mayer H: Chromatinremodeling factors allow differentiation of bone marrow cells into insulin-producing cells. Stem Cells 24: 2858-2867, 2006.

13. Phuc PV, Nhung TH, Loan DT, Chung DC and Ngoc PK: Differentiating of banked human umbilical cord blood-derived mesenchymal stem cells into insulin-secreting cells. In Vitro Cell Dev Biol Anim 47: 54-63, 2011.

14. Chao KC, Chao KF, Fu YS and Liu SH: Islet-like clusters derived from mesenchymal stem cells in Wharton's Jelly of the human umbilical cord for transplantation to control type 1 diabetes. PLoS One 3: e1451, 2008.

15. Shiroi A, Ueda S, Ouji Y, et al: Differentiation of embryonic stem cells into insulin-producing cells promoted by Nkx2.2 gene transfer. World J Gastroenterol 21: 4161-4166, 2005.

16. Segev H, Fishman B, Ziskind A, Shulman M and Itskovitz-Eldor J: Differentiation of human embryonic stem cells into insulinproducing clusters. Stem Cells 22: 265-274, 2004.

17. Bai L, Meredith G and Tuch BE: Glucagon-like peptide-1 enhances production of insulin in insulin-producing cells derived from mouse embryonic stem cells. J Endocrinol 186: 343-352, 2005.

18. Hui H, Yu R, Bousquet $C$ and Perfetti R: Transfection of pancreatic-derived beta-cells with a minigene encoding for human glucagon-like peptide-1 regulates glucose-dependent insulin synthesis and secretion. Endocrinology 143: 3529-3539, 2002.

19. Li L, Li F, Qi H,Feng G, Yuan K, Deng H and Zhou H: Coexpression of Pdx1 and betacellulin in mesenchymal stem cells could promote the differentiation of nestin-positive epithelium-like progenitors and pancreatic islet-like spheroids. Stem Cells Dev 17: 815-823, 2008.

20. Vaca P, Berná G, Araujo R, Carneiro EM, Bedoya FJ, Soria B and Martín F: Nicotinamide induces differentiation of embryonic stem cells into insulin-secreting cells. Exp Cell Res 314: 969-974, 2008.

21. Cho YM, Lim JM, Yoo DH, et al: Betacellulin and nicotinamide sustain PDX1 expression and induce pancreatic beta-cell differentiation in human embryonic stem cells. Biochem Biophys Res Commun 366: 129-134, 2008.

22. Hisanaga E, Park KY, Yamada S, et al: A simple method to induce differentiation of murine bone marrow mesenchymal cells to insulin-producing cells using conophylline and betacellulindelta4. Endocr J 55: 535-543, 2008.

23. Ye DZ, Tai MH, Linning KD, Szabo C and Olson LK: MafA expression and insulin promoter activity are induced by nicotinamide and related compounds in INS-1 pancreatic beta-cells. Diabetes 55: 742-750, 2006.

24. Beattie GM, Montgomery AM, Lopez AD, et al: A novel approach to increase human islet cell mass while preserving beta-cell function. Diabetes 51: 3435-3439, 2002.

25. Habener JF, Kemp DM and Thomas MK: Minireview: transcriptional regulation in pancreatic development. Endocrinology 146: 1025-1034, 2005.

26. Miyazaki S, Yamato E and Miyazaki J: Regulated expression of pdx-1 promotes in vitro differentiation of insulin-producing cells from embryonic stem cells. Diabetes 53: 1030-1037, 2004.

27. Baertschiger RM, Bosco D, Morel P, Serre-Beinier V, Berney T, Buhler LH and Gonelle-Gispert C: Mesenchymal stem cells derived from human exocrine pancreas express transcription factors implicated in beta-cell development. Pancreas 37: 75-84, 2008.

28. Nelson SB, Schaffer AE and Sander M: The transcription factors Nkx6.1 and Nkx6.2 possess equivalent activities in promoting beta-cell fate specification in $\mathrm{Pdx} 1^{+}$pancreatic progenitor cells. Development 134: 2491-2500, 2007. 